\title{
Drug Reaction with Eosinophilia and Systemic Symptoms Syndrome: Quick Defervescence with Early Steroid Therapy
}

\author{
Malav Thakkar ${ }^{1}$, Surjeet Kumar ${ }^{2}$, Chenniganahosahalli Revaiah Vishwa ${ }^{3}$, Suresh K Angurana ${ }^{4}$, Lokesh Saini ${ }^{5}$, \\ Keshavamurthy Vinay ${ }^{6}$
}

\begin{abstract}
Drug reaction with eosinophilia and systemic symptoms (DRESS) syndrome or drug-induced hypersensitivity syndrome or anticonvulsant hypersensitivity syndrome is a delayed hypersensitivity drug rash that occurs 2-6 weeks after starting anticonvulsants, sulphonamides, or antibiotics. It is characterized by a triad of fever, rash, and systemic manifestations with an overall mortality of $10 \%$. We reported a $1 \frac{1}{2}$-year-old girl who was a known case of structural West syndrome and was on phenobarbitone, valproate, and levetiracetam. She presented with fever, rash, liver dysfunction, and eosinophilia. Diagnosis of DRESS was considered and was treated with IV methylprednisolone following which she showed rapid defervescence, healing of rash, and improvement in liver dysfunction within the next 4 days. Rapid response to specific therapy made us report this case.

Keywords: Anticonvulsant, Eosinophilia, Liver dysfunction, Steroids.

Journal of Postgraduate Medicine, Education and Research (2021): 10.5005/jp-journals-10028-1440
\end{abstract}

\section{INTRODUCTION}

Drug reaction with eosinophilia and systemic symptoms (DRESS) syndrome is a rare, idiosyncratic, delayed hypersensitivity drug reaction that occurs classically 2-6 weeks after starting anticonvulsants (carbamazepine, phenytoin, phenobarbitone, oxcarbazepine, lamotrigine), other drugs (sulphonamides, minocycline, allopurinol, dapsone, sulfasalazine, etc.), and antibiotics and it is potentially life-threatening. ${ }^{1-3}$ The estimated incidence of DRESS syndrome ranges from 1 in 1,000 to 1 in 10,000 drug exposure (1 in 1,500 new users of phenytoin and carbamazepine) and mortality as high as $10 \% .^{2}$ The treatment includes withdrawal of offending medication, supportive care, and systemic steroids. ${ }^{4}$

The reason for reporting this case is to describe a child with characteristic features of DRESS syndrome due to antiepileptic drugs and who responded quickly to pulse methylprednisolone. There are only limited reports in the literature where children with DRESS syndrome were treated with pulse methylprednisolone. The clinicians should be aware of this complication as early recognition and prompt management may be life-saving.

\section{Case Description}

We report a 11/2-year-old girl, known case of West syndrome receiving phenobarbitone ( $5 \mathrm{mg} / \mathrm{kg} /$ day), valproate $(40 \mathrm{mg} / \mathrm{kg} /$ day), and levetiracetam ( $20 \mathrm{mg} / \mathrm{kg} /$ day) for the last 6 months. She developed sudden onset of febrile illness with generalized erythematous, maculopapular, pruritic rash involving face, trunk, and limbs (Figs 1A and B). In 3-4 days, the rash became confluent. She also had generalized edema which was more pronounced over face and trunk (Fig. 1A) and dorsum of hands and feet.

Laboratory investigations revealed eosinophilia $\left(1,690 / \mathrm{mm}^{3}\right)$, transaminitis [AST $197 \mathrm{U} / \mathrm{L}$ (normal: 13-35 U/L) and ALT $192 \mathrm{U} / \mathrm{L}$ (normal: 5-45 U/L)], coagulopathy (INR 2.3), normal renal functions, and elevated CRP [19.5 mg/dL (normal: $<0.5 \mathrm{mg} / \mathrm{dL}$ )]. Serology for EBV, Mycoplasma, CMV, HAV, HBV, HCV, and Chlamydia were
${ }^{1}$ Department of Pediatrics, Advanced Pediatrics Centre, Postgraduate Institute of Medical Education and Research, Chandigarh, India

${ }^{2-4}$ Division of Pediatric Critical Care, Department of Pediatrics, Advanced Pediatrics Centre, Postgraduate Institute of Medical Education and Research, Chandigarh, India

${ }^{5}$ Division of Pediatric Neurology, Department of Pediatrics, Advanced Pediatrics Centre, Postgraduate Institute of Medical Education and Research, Chandigarh, India

${ }^{6}$ Department of Dermatology, Venereology, and Leprosy, Postgraduate Institute of Medical Education and Research, Chandigarh, India

Corresponding Author: Suresh K Angurana, Division of Pediatric Critical Care, Department of Pediatrics, Advanced Pediatrics Centre, Postgraduate Institute of Medical Education and Research, Chandigarh, India, Phone: +91 9855373969, e-mail: sureshangurana@ gmail.com

How to cite this article: Thakkar M, Kumar S, Vishwa CR, et al. Drug Reaction with Eosinophilia and Systemic Symptoms Syndrome: Quick Defervescence with Early Steroid Therapy. J Postgrad Med Edu Res 2021;55(2):77-78.

Source of support: Nil

Conflict of interest: None

negative and blood culture was sterile. The RegiSCAR score was 7. The treatment included discontinuation of phenobarbitone and valproate, continuation of levetiracetam, and addition of clobazam. In view of systemic involvement, she received intravenous methylprednisolone ( $30 \mathrm{mg} / \mathrm{kg} /$ day) for 3 days followed by oral prednisolone ( $2 \mathrm{mg} / \mathrm{kg} /$ day). There was rapid defervescence, healing of rash (Fig. 1C), and improvement in liver dysfunction within next 5 days.

\section{Discussion}

The common anticonvulsant drugs leading to DRESS syndrome are carbamazepine, phenytoin, phenobarbitone, oxcarbazepine, 

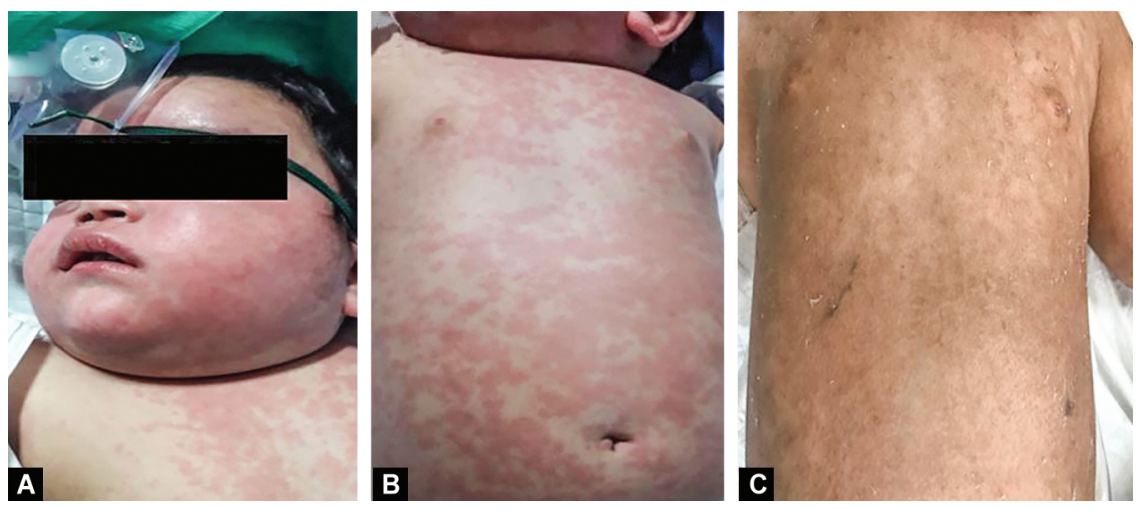

Figs $1 \mathrm{~A}$ to $\mathrm{C}$ : Clinical image showing rash involving face and trunk on day 1 of admission (A and B) and day 14 of admission (C)

and lamotrigine. The pathogenesis of DRESS syndrome is not completely understood and it is postulated that there is an interaction between genetic predisposition, abnormalities in metabolic pathways leading to accumulation of drug metabolites, and drug-virus interactions leading to reactivation of HHV-6 and HHV-7, EBV, and CMV. ${ }^{1-3}$

It presents as a spectrum from mild rash with hematological manifestation which response to the withdrawal of offending drug to severe multiorgan involvement requiring immunosuppression. It is characterized by a triad of fever, rash, and hepatitis. The rash is initially located over the face, upper trunk, and arms in form of diffuse exanthem of morbilliform pruritic papules to plaques. Exfoliation is uncommon and so is the mucous membrane involvement. Eosinophilia ( $\geq 500 / \mu \mathrm{L})$ and atypical lymphocytosis are common features but not always present. There may be facial edema, cervical lymphadenopathy, pharyngitis, and malaise. Multisystemic involvement in form of hepatitis (90\%) (ranging from a mild elevation of liver transaminase values to frank hepatic failure), interstitial nephritis (9\%), pneumonitis (5\%), myocarditis, shock, and encephalitis can occur in severe disease.

Diagnosis of DRESS syndrome is difficult to establish, and it requires a high level of suspicion as well as ruling out other etiologies. ${ }^{4}$ These include infectious disease (e.g., viral exanthemas, staphylococcal and streptococcal toxic shock syndromes, meningococcemia), non-infectious drug eruptions (e.g., StevensJohnson syndrome, toxic epidermal necrolysis), autoimmune disease (e.g., Kawasaki disease, hypereosinophilic syndrome), and neoplastic diseases (e.g., leukemia cutis, mycosis fungoides). Depending on the specific organs involved, the differential diagnosis also includes viral hepatitis (liver), glomerulonephritis, vasculitides, pre- and postrenal causes of acute kidney injury (kidney), Kawasaki disease and eosinophilic myocarditis (heart), parasitic infection (gastrointestinal tract), and bacterial, viral, and fungal pathogens (lungs). There are no available pathognomonic signs or diagnostic tests. The diagnosis is clinical and established by taking into account drug exposure in the appropriate clinical setting and latency between drug exposure and symptom onset. Re-challenging with the causative drug has been the gold standard to diagnose drug eruptions but should not be used in suspected DRESS cases as it may be life-threatening. The most commonly used diagnostic criteria included a scoring system proposed by the RegiSCAR group. ${ }^{1}$ This scoring system comprises the major features of DRESS syndrome, giving each item a score of minus one point, zero points, one point, or two points. The diagnosis of DRESS syndrome is then made based on the total score: $<2$ points: no case; $2-3$ points: possible case; $4-5$ points: probable case; $>5$ points: definite case. In the index case, she had fever, lymphadenopathy, eosinophilia, skin rash ( $>50 \%$ of BSA), and organ dysfunction (liver dysfunction). So, the RegiSCAR score was 7 making her a definite case of DRESS syndrome.

Treatment of DRESS syndrome includes the immediate withdrawal of inciting drugs. Cross-sensitivity of barbiturates and carbamazepine with phenytoin has been observed. In such a setting, the drugs that can be safely used to control seizures are levetiracetam, valproate, and gabapentin. The systemic corticosteroids are the mainstay of treatment for a patient with DRESS syndrome. ${ }^{5}$ IVIG is another treatment option used in various case reports yielding conflicting results. ${ }^{6}$ In severe and corticosteroid-resistant cases, more potent immunosuppressants including cyclosporine, azathioprine, rituximab, infliximab, and mycophenolate have been used, sometimes alongside adjunctive treatment with IVIG and plasmapheresis. ${ }^{3} \mathrm{~N}$-Acetyl cysteine (NAC), which acts as a detoxifying drug, can also be used in DRESS syndrome. In addition to definite treatment, supportive care is also important to support involved organ dysfunction.

\section{Conclusion}

Clinicians should be aware of this potentially fatal cutaneous drug reaction and common medications implicated in the causation of DRESS syndrome. The prompt withdrawal of the offending drugs and early introduction of systemic steroids in severe cases may lead to a better outcome.

\section{References}

1. Cho YT, Yang CW, Chu CY. Drug reaction with eosinophilia and systemic symptoms (DRESS): an interplay among drugs, viruses, and immune system. Int J Mol Sci 2017;18(6):1243. DOI: 10.3390/ ijms18061243.

2. De A, Rajagopalan M, Sarda A, et al. Drug reaction with eosinophilia and systemic symptoms: an update and review of recent literature. Indian J Dermatol 2018;63(1):30. DOI: 10.4103/ijd.IJD_582_17.

3. Mori F, Caffarelli C, Caimmi S, et al. Drug reaction with eosinophilia and systemic symptoms (DRESS) in children. Acta Biomedica, Mattioli 1885 2019;90:66-79.

4. James BDEFJ, Sammour BDEYM, Virata ADEFGAR, , Nordin ABDEF TA, Dumic Corresponding Author I, Dumic I. Drug Reaction with Eosinophilia and Systemic Symptoms (DRESS) Syndrome Secondary to Furosemide: Case Report and Review of Literature. 2018.

5. Descamps V, Ben Saïd B, Sassolas B, et al. Prise en charge du drug reaction with eosinophilia and systemic symptoms (DRESS). Ann Dermatol Venereol 2010;137(11):703-708. DOI: 10.1016/j. annder.2010.04.024.

6. Janela B, Janela B, Tetart F, et al. Poor benefit/risk balance of intravenous immunoglobulins in DRESS. Arch Dermatol 2012;148(4):543-544. DOI: 10.1001/archderm.148.4.dlt120002-c. 